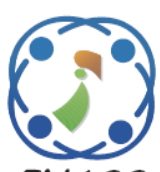

\title{
Detection of Breast Cancer on Magnetic Resonance Imaging Using Hybrid Feature Extraction and Deep Neural Network Techniques
}

\author{
Nagaraja Rao Pamula Pullaiah ${ }^{1 *}$ \\ Dorai Venkatasekhar ${ }^{2}$ \\ Balaraj Sudhakar ${ }^{1}$ \\ Padarthi Venkatramana ${ }^{3}$ \\ ${ }^{I}$ Department of Electronics \& Communication Engineering, Annamalai University, India \\ ${ }^{2}$ Department of Information Technology, Annamalai University, India \\ ${ }^{3}$ Department of Electronics \& Communication Engineering, Sree Vidyanikethan Engineering College, India \\ * Corresponding author’s Email: Nagaraj9s@ gmail.com
}

\begin{abstract}
Breast cancer is one of the most occurring cancers in women due to the uncontrolled growth of abnormal cells in the lobules or milk ducts. The treatment for the breast cancer at an early stage is important using Magnetic Resonance Imaging (MRI) which effectively measures the size of the cancer and also checks tumors in the opposite breast. The deposition of calcium components on the breast tissue is known as micro-calcifications. The calcium salts deposited in the breast are involved with the cancer and were not diagnosed accurately due to the low effectiveness of existing imaging technique namely Haralick feature extraction technique. The MRI breast cancer diagnosis creates problems during classification of breast image and leads to misclassifications, such as unidentified calcium deposits in the existing K-Nearest Neighbour (KNN) classifier. The misclassification issues are overcome by an accurate classification and identification of calcium salts and checks whether deposited salt on breast tissue is involved with cancer or not. Initially, Contrast-Limited Adaptive Histogram Equalization (CLAHE) is used to remove the unwanted noise in the MRI and Morphological, Multilevel Otsu's Thresholding and region growing techniques perform segmentation to mask unwanted breast tissues. The proposed Hybrid LOOP Haralick feature extraction technique is developed by combining the both Local Optimal Oriented Pattern (LOOP) and Haralick texture feature and the hybrid parameters are applied to the Stacked Auto Encoder based (SAE) to classify the breast MRI image as a Malignant or Benign. The performance of the proposed hybrid LOOP Haralick feature extraction shows significant accuracy improvement of $3.83 \%$ when compared to the Haralick feature extraction technique.
\end{abstract}

Keywords: Breast cancer, Contrast-limited adaptive histogram equalization, Hybrid parameters, Magnetic resonance imaging, Multilevel Otsu's thresholding, Stacked auto encoder.

\section{Introduction}

Breast cancer is the most common invasive cancer in women and the second leading cause of cancer death after lung cancer. The MRI scanning technology is commonly recommended diagnose treatment for women due its high sensitivity to detect invasive malignancies [1]. The Breast MRI usage has increased its prominence up to $20-25 \%$ as due to its effective diagnosis at the early stage of the disease [2]. The properties of the breast cancer anomalies are diagnosed at its early stage to control spread of cancer cells and the human visual perception is quite difficult to determine the abnormalities during misclassification [3]. The detection of malignancy risk in breast image is studied by the department of American College of Radiology (ACR) using Breast Imaging Reporting and Data System (BI-RADS) dataset [4]. The imaging techniques used in the existing researches are time consuming and complex task, which requires correlation to multi-parametric and interpretation of data to accurate classification [5, 6]. The radiologist uses BI-RADS dataset for clinical assistance to overcome the aforementioned problems in the existing methods. The advantages of using MRI are its feasible property in a system and obtains high specificity and sensitivity for spatial and 
temporal imaging techniques [7, 8]. The calcium components deposited on the breast tissues are not diagnosed in the MRI images that create a misclassification issue in diseased regions $[9,10]$.

The proposed research initially performs a preprocessing technique known as CLAHE to remove unwanted noises and the region growing technique perform masking for the pre-processed MRI breast images. The aim of this research is to determine appropriate parameters from hybrid LOOP Haralick texture feature extraction techniques and the extracted features are applied to the SAE classifier for identifying abnormal breast tissues. Based on the hybrid parameters, the classification is performed using SAE which is constructed based on Deep Neural Network (DNN) classifies breast MRI image is a Malignant or Benign.

This paper is organized as follows: In section 2, various researches based on the detection of breast cancer are reviewed. The proposed system is explained in Section 3 and Section 4 illustrates the quantitative analysis and comparative analysis of the proposed system with the existing techniques. The conclusion of this research study is made in section 5 .

\section{Literature review}

Various methodologies were analysed for breast cancer detection using MRI image to diagnose and control the spread of cancer cells as earlier as possible. A review of existing methods undergone in determining breast cancer using medical imaging techniques is explained in this section with its brief advantages and limitations.

Wengert [11] Evaluated impact of the Kaiser score on clinical decision-making in BI-RADS 4 mammographic calcifications in breast MRI. The main objective of this research was to investigate the Kaiser score that would not unnecessarily degrade breast lesions and mammographic calcifications. The examinations are performed for different levels of classification to examine Kaiser score independently. The machine learning tool was used for clinical decision making to provide the malignancy probabilities. The performance of the proposed method was assessed diagnostically by analysing the inter-reader agreement using Receiver Operating Characteristics (ROC) and calculated Cohen's kappa coefficients. The proposed model classified breast cancer MRI lesion and removed the calcification on breast biopsies. The mammographic calcifications diagnosing was a challenging task on MRI due to the analysis of the inter-reader agreement to classify the malignant and benign breast lesions.
Ji [12] diagnosed breast cancer using Artificial intelligence on MRI. The evaluation of machine learning diagnosed the disease in advance using artificial intelligence methods. The classification of breast cancer disease was done by Support Vector Machine (SVM) that classifies breast MRI into malignant and benign lesions. The developed model performed computer analysis on consecutive Dynamic Contrast Enhanced MRI (DCE-MRI) studies. The radiomic features were extracted automatically from the segmented lesions and that were given to the SVM. The ROC was obtained on the independent consecutive clinical dataset which distinguishes breast lesions as benign or malignant. An advantage of using the model was that both morphological and kinetic information was obtained from MRI examination. From the results it was analysed that the single vendor performed both independent testing and training for unique patient patterns but it was failed to evaluate external vendor images.

Lunkiewicz [13] developed Inter observer variability to determine the malignancy for fifth edition BI-RADS MRI descriptors in mass breast lesions. The developed model performed Non-Mass Enhancement (NME) for the breast MRI, thereby extracting common features of Ductal Carcinoma In Situ. The developed model introduced an enhancement technique called clustered ring that significantly segmented the newly distributed patterns of the predicted malignancy among the NME descriptors. An advantage of the developed model was that the combined interpretation skills showed high-quality improvement in the detection rate of the tumour. However, the developed model included only MRI lesions subjected to higher inter-observer variability which was not having many distinct lesions to evaluate the size of the breast lesion.

Menezes [14] developed a dynamic contrastenhanced breast MRI at 7T and 3T for lesion identification to perform classification on BI-RADS dataset. The developed model used BI-RADS dataset for classification and it was evaluated by radiologists to identify the image quality and breast lesions. The developed model extracted seventeen Histopathological features related to malignant lesions from the detected strength of field. The BIRADS classifications showed improvement in specificity, sensitivity, size of lesions and ContrastEnhancement to Noise Ratios. The results showed that the dynamic series of the developed model obtained image quality of $7 \mathrm{~T}$, where $1 \mathrm{~T}-3 \mathrm{~T}$ from the BI-RADS lesion belonged to benign and 4T-5T belonged to malignant. However, similar lesions 
present in few patients leads to improper accuracy evaluation among benign and high risk lesions.

Gweon [15] executed management for BI-RADS category into 3 lesions detected in preoperative breast MR imaging of breast cancer patients. The developed model performed NME which was based on stippled and homogenous pattern that isolated dominant focus of malignancy level in an image. Based on the multiple bilateral foci, malignant and benign breast cancer cases were distinguished. However, the developed model used less number of malignant cases which was difficult for accurate classification. Amico [16] developed KNN approach for differentiating malignant from benign enhancing foci on BI-RADS dataset that consisted of breast MRI. The developed model used Semeion's Training With Input Selection and Testing (TWIST) algorithm aimed to solve the features selection and training splitting problems. The KNN operated fast and robust classification of malignant and benign classes resulted best performance. However, the variability associated to differences in MRI sequences, devices and contrast agents needed to be addressed.

\section{Proposed methodology}

The block diagram of the proposed LOOP Haralick feature extraction model is presented in the Fig. 1. The block diagram consists of BI-RADS dataset, Pre-processing stage, Region growing, Multilevel Otsu thresholding and Morphological operator, Feature Extraction and classification of malignant and benign breast image using DNN.

\subsection{BI-RADS dataset}

BI-RADS are a dataset used for the prediction of benign or malignant in the mass lesion of MRI scans which consist of patients' attributes and their ages [17]. The properties of BIRADs dataset are shown in the Table 1 and the input images from BIRADS dataset is presented in Fig. 2.

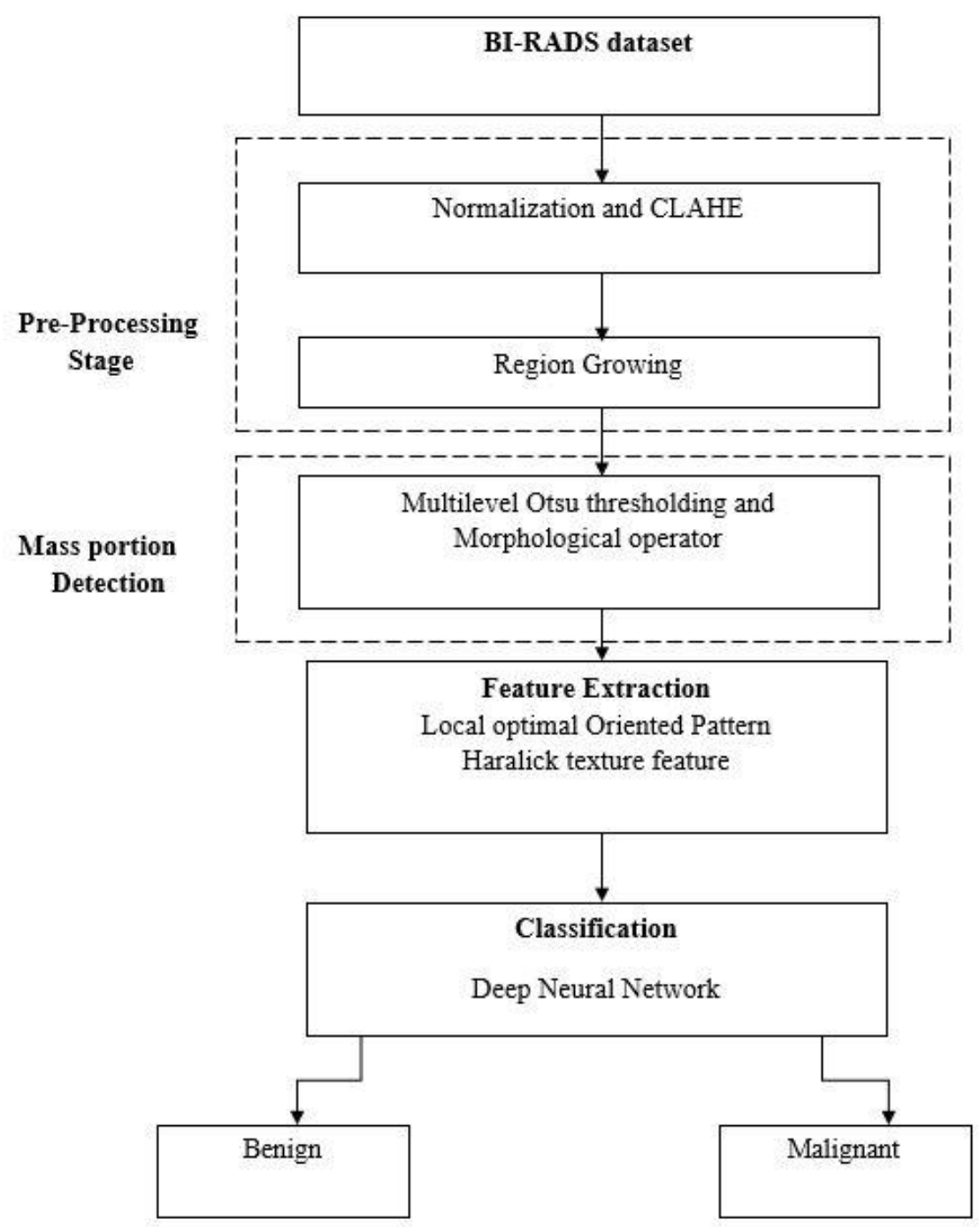

Figure. 1 Block diagram of the proposed methodology 
Table 1. BI-RADS dataset's properties

\begin{tabular}{|c|c|}
\hline \multicolumn{2}{|c|}{ American college of radiology } \\
\hline Age range & $13-92$ years \\
\hline Period (Year) & 2009 to 2017 \\
\hline Overall images & 2330 \\
\hline
\end{tabular}

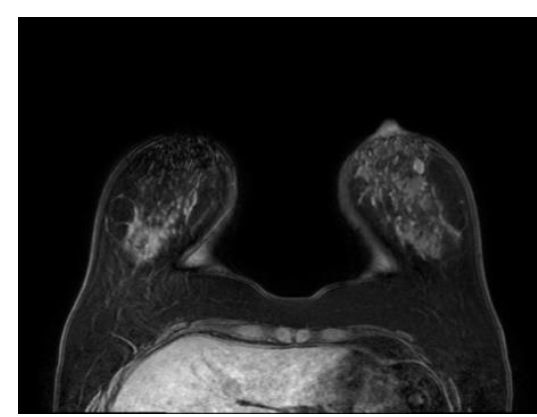

(a)

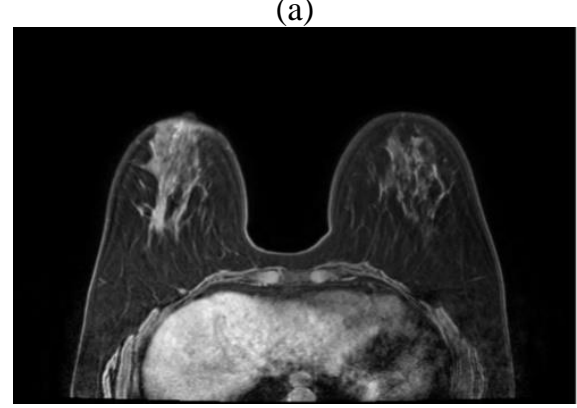

(b)

Figure. 2 Input images from BIRADS dataset: (a)-benign and (b) malignant)

The instance ranging from 1 to 5 is rated as benign and the increase in the range above 5 is considered as malignancy by the physicians. The sensitivity and specificity of the BI-RADS are calculated based on the level of malignancy and benign.

The BI-RADS dataset has,

- 700 plus clinical images

- Ultrasound, MRI, and mammography outcome monitoring system

- MRI breast implant descriptors.

- Follow-up and outcome monitoring includes mammography, ultrasound and MRI

- Updated breast composition descriptors

\subsection{Pre-processing}

The MRI images usually consist of dark large area which is not a breast tissue and it requires an accelerating process to remove those unwanted lesions from MRI images to avoid misclassification. The noise is created when magnetic fields are cycled ON and OFF during MRI scanning and these unwanted noises and dark areas are removed using normalization and CLAHE process.

\subsubsection{Normalization and CLAHE}

The MRI image generated by MRI scanners includes noise and non-uniform intensity distribution, so the image normalization technique is used to solve such problem during MRI normalization. MRI normalization is challenging task due to the requirement of intensity and mapping values to remove unwanted noise in MRI. The image normalization process used to change the normal intensity values of pixels to more familiar intensity values of pixels for normal sensing. Similarly, the CLAHE algorithm used widely to enhance the contrast in medical images, but it concentrates only tile region of the image instead of considering whole image. The enhancement of each tile contrast is achieved the output matches approximately in the specified region using the distribution parameter. The bilinear interpolation is used to remove the neighboring tiles to eliminate artificial boundaries. The steps involved in pre-processing of MRI images using CLAHE are as follows

The CLAHE uses two main parameters to control the image quality, such as block size and clip limit. The process of CLAHE is described as follows:

- Initially, the original intensity image $(I)$ is divided into non-overlapping contextual regions. In that case, the total amount of image tiles is equal to $M \times N$.

- For each contextual region, the histogram is calculated with respect to the gray levels exist in the array image.

- The clipped histogram and gray level mapping are generated in CLAHE. The pixels are equally separated at each gray level and Eq. (1) expresses the average number of pixels.

$$
N_{\text {avg }}=\left(N_{r} X \times N_{r} Y\right) / N_{\text {gray }}
$$

Where, $N_{\text {avg }}$ represents the average amount of pixel and $N_{\text {gray }}$ represents the amount of gray level in the contextual region. The amount of pixels present in $X$ and $Y$ dimension are $N_{r} X$ and $N_{r} Y$ respectively. The clip limit is expressed in Eq. (2).

$$
N_{C L}=N_{\text {clip }} \times N_{\text {avg }}
$$

Where, $N_{C L}$ represents the actual clip limit and $N_{\text {clip }}$ is normalized clip limit in $[0,1]$ range. The preprocessed image using CLAHE is presented in the Fig. 3. 


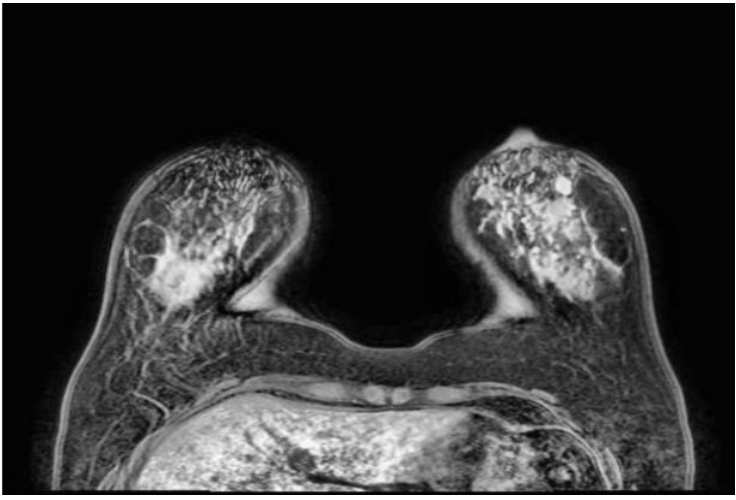

(a)

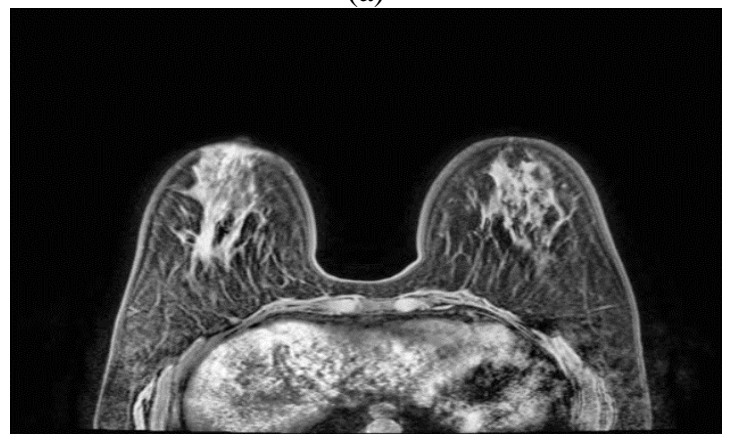

(b)

Figure. 3 Enhanced image using CLAHE: (a) benign and (b) malignant

\subsubsection{Region growing}

The region growing is also known as simple region based segmentation technique used for performing image's pixel segmentation which is involved in the selection of initial seed points. The neighbourhood pixels are examined initially at the seed point to determine whether the pixel neighbour should be added to that masked breast region or not. The research work performs region growing in each of the iterations to identify the mask template. The masking preserves breast tissues especially nipple regions and applies to all types of breast tissue for accurate determination of cancer. The breast mask segmentation is performed by computing both $\mathrm{T} 1 \mathrm{w}$ and $\mathrm{T} 2 \mathrm{w}$ images and steps of breast mask segmentation given as follows,

Step 1: The resampled T2w image is matched by resolution of T1w image.

Step2: The voids are eliminated subsequently during foreground segmentation of $\mathrm{T} 2 \mathrm{w}$ image

Step 3: The maximum intensity is achieved by matching the rescaling of $\mathrm{T} 1 \mathrm{w}$ image with intensity of the closed T2w image.

Step 4: The segmentation of foreground image is obtained from the background image and the output image of region growing is presented in the Fig. 4.

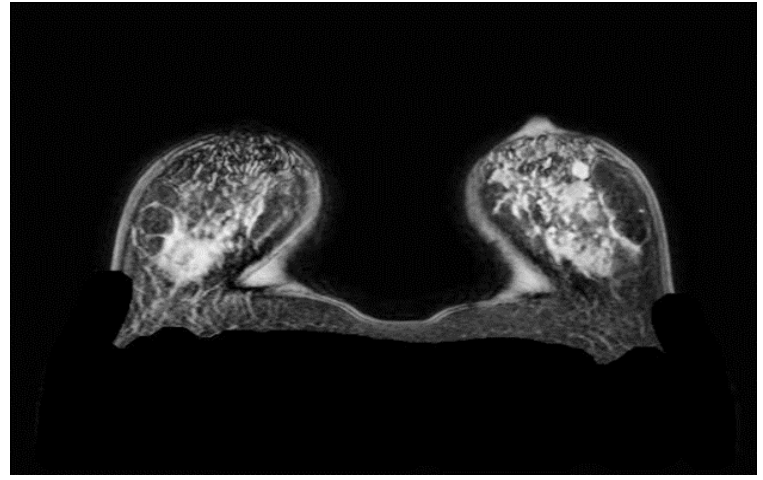

(a)

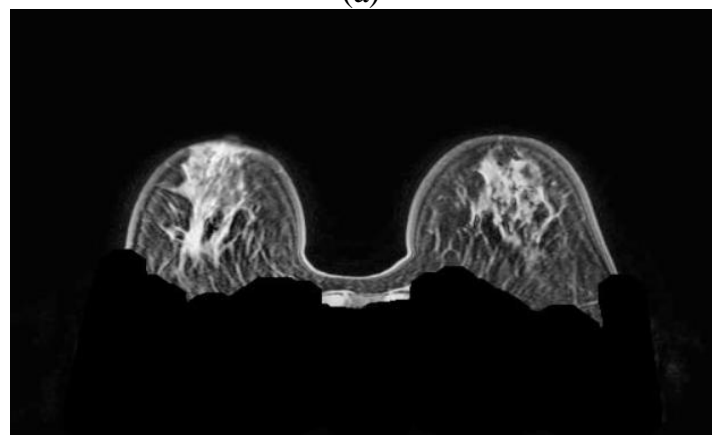

(b)

Figure. 4 Image obtained after using region growing: (a) benign and (b)malignant

\subsection{Mass portion detection using multilevel Otsu thresholding and morphological operator}

The segmentation of masking region is done by using a region growing technique, but still the unwanted regions from the segmented breast MRI needed to be removed. The Morphology operation is used in this research to remove unwanted regions based on the shapes and sizes of an input image. The segmented mask region with structural element is given as input image to morphological operations and it produces the same size output image without losing any important qualities. The morphological operation is performed for each pixel of an input image that corresponds to the neighborhood pixels. The shape and size of an image are chosen based on the neighborhood pixels and morphological operation is performed to construct specific shapes for an input image. Similarly, multilevel - Otsu threshold algorithm performs pixel separation of an input image into different classes that separated gray levels based on the intensity values. The threshold values of gray level based images are calculated using multiOtsu that determines classes of desired number. The main reason of using Multilevel Otsu thresholding and Morphological operator techniques is to eliminate unwanted regions, even after masking is achieved using region growing. The image obtained after segmentation is presented in Fig. 5. 


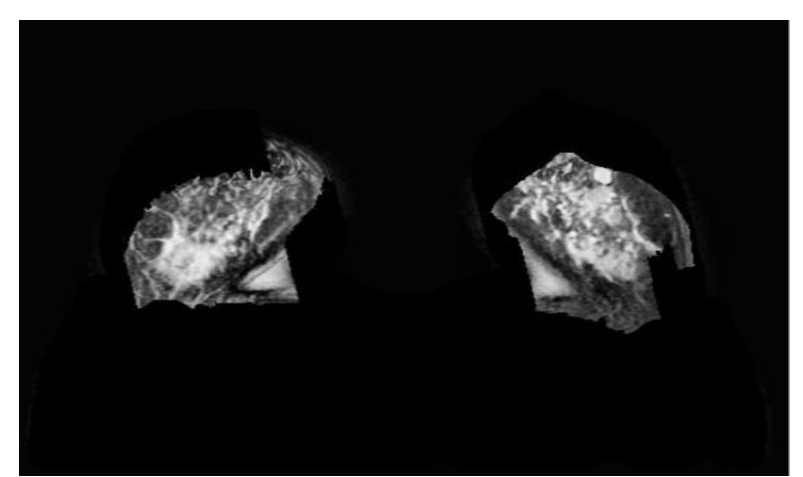

(a)

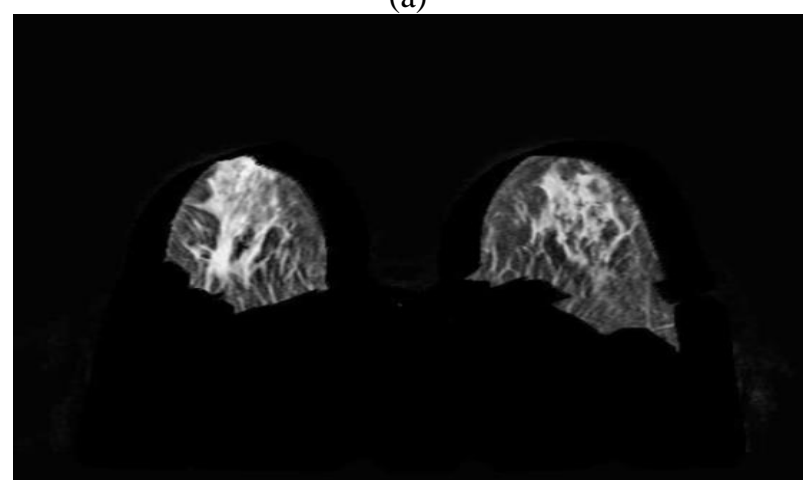

(b)

Figure. 5 Image obtained after segmentation: (a) benign and (b) malignant

\subsection{Hybrid feature extraction}

After performing morphological operation, extraction of feature from the segmented region is important for breast cancer detection. The input data transformation into set of features is known as feature extraction. The extracted features are chosen from a set of features that consists of relevant input information of the image. The features perform desired task and reduces data representation for the full size input. The extracted features usually consist of information related to the texture, shape or context or the gray shade.

\subsubsection{Local optimal oriented pattern}

The major disadvantage of Local Derivative Pattern (LDP) and Local Binary Pattern (LBP) feature extraction technique were that the empirical value assigned to the threshold variable restricts allowance of bits thereby reduces the number of possible words. The LOOP is used to overcome the aforementioned problems, which amalgams LDP and LBP to preserve strength in each case. The LOOP feature parameters are shown in the Table 2.

The LDP uses 8 Kirsch masks according to the eight neighboring pixels direction $n=0,1 \ldots 7$, which measures the variations in the intensity strength in respective directions. Similarly, the LBP
Table 2. LOOP feature parameters

\begin{tabular}{|c|c|}
\hline Symbols & Description \\
\hline$i_{n}$ & Intensity of an image \\
\hline $\mathrm{I}$ & Image \\
\hline$\left(x_{c}, y_{c}\right)$ & Pixel dimension \\
\hline$i_{c}$ & Central pixel of an image \\
\hline $3 \times 3$ & Intensity of pixel $(\mathrm{n}=0 \ldots 7)$ \\
\hline
\end{tabular}

also indicates variations occurring in the intensity of neighboring pixels, where the value of Kirsch output is employed by assigning computed decimal-tobinary weights. The eight responses to obtain Kirsch masks $m_{n}$ are essential from $n=0 \ldots .$. are essential to achieve the pixel intensity of the images. Each of the pixels are allotted with respective digits between 0 to 7 as the magnitude called $m_{n}$ among the eight kirsch mask outputs, which is expressed in Eqs. (3) and (4).

$$
\begin{gathered}
\operatorname{LOOP}\left(x_{c}, y_{c}\right)=\sum_{n=0}^{7} s\left(i_{n}-i_{c}\right) \cdot 2^{w_{n}} \\
s(x)=\left\{\begin{array}{cc}
1 & \text { if } x \geq 0 \\
0 & \text { Otherwise }
\end{array}\right\}
\end{gathered}
$$

Thus, the LOOP descriptor encodes rotation invariance into the main formulation. Moreover, the proposed LOOP algorithm also mitigates the empirical assignment parameter $k$ when compared with the traditional LDP method.

\subsubsection{Haralick texture feature}

Haralick texture features are derived from GLCM $2 \mathrm{D}$ formulation which is constructed in matrix format has 26 different offsets matched with a single matrix. From this matrix, 16Haralick features are derived. Among 16 features the proposed method considers only necessary 4 features for classification such as contrast, correlation, Entropy and variance.

\section{Contrast}

The difference in the intensity of an image based on local region is defined as contrast. The mathematical expression of contrast is given in Eq. (5).

$$
\begin{aligned}
& \text { Contrast }=\sum_{n=0}^{N_{g}-1} n^{2}\left\{\sum_{i=1}^{N_{g}} \sum_{j=1}^{N_{g}} p(i, j)\right\} \\
& |i-j|=n
\end{aligned}
$$

\section{Correlation}

Correlation measures the linear dependency of a gray level on the neighboring pixel of an image. 
The mathematical expression of Correlation is given in Eq. (6)

$$
\text { Correlation }=\frac{\sum_{i} \sum_{j}(i j) p(i, j)-\mu_{x} \mu_{y}}{\sigma_{x} \sigma_{y}}
$$

Where $\mu_{x}, \mu_{y}$ are the mean values $\sigma_{x}, \sigma_{y}$ Referred referred as standard deviations and $p_{x}, p_{y}$ are the partial probabilities for the density functions.

\section{Energy}

Energy features are derived based on the Angular Second Moment (ASM). ASM is the measure of local uniformity in gray levels. The mathematical expression for energy and ASM are given in Eqs. (7) and (8).

$$
\begin{gathered}
\text { Energy }=\sqrt{A S M} \\
A S M=\sum_{i} \sum_{j} p_{d}^{2}(i, j)
\end{gathered}
$$

Where $p_{d}$ is the probability density function.

\section{Variance feature}

The variance refers to the calculation of threshold which is dependent on probability density function of a particular distribution. The mathematical expression for Variance feature is given in Eq. (9).

$$
\sum_{i} \sum_{j}(i-\mu) 2 p(i, j)
$$

The following steps are executed for the proposed Hybrid LOOP Haralick feature extraction technique.

Step 1: Initially, the texture analysis is performed by using the Haralick features that maps the graylevels in an original image, which expressed in Eq. (10).

$$
\psi:[a, b]^{M X K} \rightarrow[1, N]^{M \times K}
$$

Step 2: The non -normalized matrix is constructed in the second step is represented in Eq. (11).

$$
\sum_{m=1}^{M} \sum_{m=1}^{M}\left\{\begin{array}{c}
X(i, j)= \\
1, \text { if } I(m, k)=i \\
1, \text { if } I\left(m+d_{x} k+d_{y}\right)=j \\
0 \quad \text { Otherwise }
\end{array}\right.
$$

Where, the non-normalized matrix parameters are shown in the Table 3.
Table 3. Parameter of Non-normalized matrix

\begin{tabular}{|c|c|}
\hline \multicolumn{1}{|c|}{$\begin{array}{c}\text { Image } \\
=(d x, d y)\end{array}$} & Displacement vector \\
\hline$x, y$ & Unit Pixels \\
\hline$M, K$ & $\begin{array}{c}\text { Maximum number of neighborhood } \\
\text { pixels }\end{array}$ \\
\hline$X(i, j)$ & Each element in GLCM \\
\hline
\end{tabular}

Step 3: Normalization is performed during GLCM construction is given in Eq. (12).

$$
P=\frac{X}{\sum_{i=1}^{N} \sum_{j=1}^{N} X(i, j)}
$$

Step 4: The last step is to perform computation for the texture features during GLCM normalization. The texture feature for Haralick can be written in general as given in Eq. (13).

$$
f=\sum_{i=a}^{A} \sum_{j=b}^{B} \phi(i, j, g(P)) \psi(p(i, j))
$$

\subsection{Stacked auto encoder based on deep neural network for classification}

The deep neural network is different from ancestral neural networks, which shows improvement in approximation properties and it has ability to represent any non-linear functions. The huge number of non-linear functions is represented in the form of layers which provides approximation of different classes. The texture features fed into deep learning methods are set as hierarchy levels of higher to the lower level features. The training of the network is performed by adjusting the weights using back propagation algorithm. In addition, the SAEs are used in the deep learning model, where SAE acts as DNN and vice versa. The combination of these two techniques achieves multi fault gearbox diagnosis. The frame work of SAE based DNN structure is presented in the Fig. 6 .

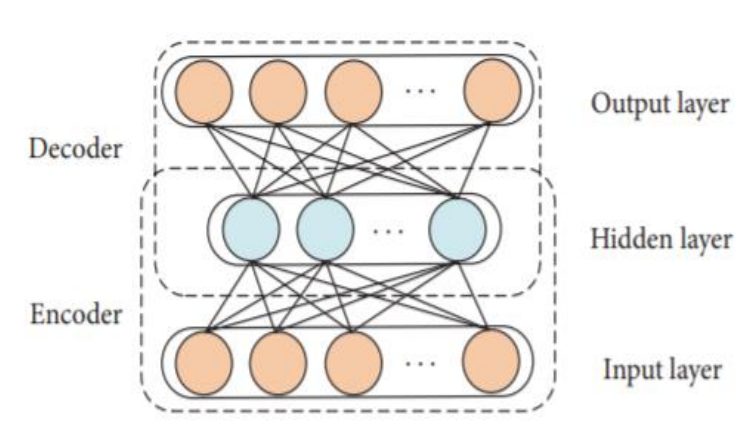

Figure. 6 Stacked auto encoder based on deep neural network 
The SAE model used to combine with the dropout model to train the weight matrix from the signals that are vibrated in the form of spectra. The training procedure of weight matrix into SAE is described as follows:

1. The vibration of the spectra signals is composed of training set $\left\{X_{i}, l_{i}\right\} M_{i}=1$

Where,

2. $M$ is number of samples. Similarly, $X_{j} \in R$ for $N \times 1$ is the ith sample having $N$ Fourier coefficients and $l_{i}$ is the health label.

3. With the help of SAE, DNNs are building and unlabeled training set by $\left\{X_{i}\right\} M_{i}=1$ using the DNN layer.

4. The weights and the parameter of SAs are updated by using the Back Propagation algorithm where the training set is labelled $\operatorname{as}\left\{X_{i}\right\} M_{i}=1$.

The SEN based on DNN classifies the BI-RADS MRI scans into Benign or malignant images. For the obtained classes, performance measures are also evaluated which is explained in the next section.

\section{Results}

The experimental setup and performance evaluation of the breast cancer image using MRI is described in this section. The proposed hybrid LOOP Haralick feature extraction is applied to BI-RADS dataset to accurately classify the MRI breast image. The specification and tools used for the proposed system is presented in the Table 4 .

The performance metrics of the proposed method is analysed in terms of accuracy, sensitivity, specificity, PPV and NPV and are explained as follows:

Accuracy: Accuracy is defined as the percentage of correctly identified breast images from the total number of breast images during classification. The mathematical expression of classification accuracy is given in Eq. (14).

$$
\text { Accuracy }=\frac{T P+T N}{T P+F P+F N+T N} \times 100
$$

Sensitivity: Sensitivity is the amount of correctly identified actual positive images during classification. The sensitivity is expressed in the Eq. (15).

$$
\text { Sensitivity }=\frac{T P}{T P+F N} \times 100
$$

Specificity: Specificity is the amount of actual negative images that are correctly identified during
Table 4. Tools and the specification of the proposed

\begin{tabular}{|c|c|}
\hline \multicolumn{2}{|c|}{ system } \\
\hline Software & MATLAB R2018a \\
\hline RAM & $16 \mathrm{~GB}$ \\
\hline Processor & i7 \\
\hline $\begin{array}{c}\text { Training and Testing } \\
\text { percentages }\end{array}$ & $70 \%$ and $30 \%$ \\
\hline
\end{tabular}

classification. The Specificity is expressed in the Eq. (16).

$$
\text { Specificity }=\frac{T N}{T N+F P} \times 100
$$

Positive Predictive Value (PPV) and Negative Predictive Value (NPV): PPV is the probability that subjects with a positive screening test truly have the disease. Negative predictive value is the probability that subjects with a negative screening test truly don't have the disease. The mathematical expression for PPV and NPV are given in Eqs. (17) and (18).

$$
\begin{aligned}
& N P V=\frac{T N}{T N+F N} \times 100 \\
& P P V=\frac{T P}{T P+F P} \times 100
\end{aligned}
$$

Where, TP, TN, FP and FN represent True Positive, True Negative, False Positive, and False Negative respectively.

\subsection{Performance analysis of the proposed method}

The performance of proposed hybrid LOOP Haralick feature extraction of the BI-RADS dataset is analysed in this section. In this study, SAE based DNN classifier is used to classify the breast MRI image as benign or malignant. In order to perform classification, a hybrid feature extraction using LOOP and Haralick features is extracted. The parameters involved in the evaluation of feature extraction are the contrast, energy, Correlation, Variance. The LOOP feature and the combination of both LOOP and Haralick features are represented in Table 5. The classification results are evaluated in terms of accuracy, sensitivity, specificity, PPV and NPV. Moreover, the textural and statistical features are evaluated to generate hybrid features. The results obtained for the parameters contrast, energy, Correlation, Variance with respect to the performance measures using DNN classifier are given as follows.

The performance measures such as Accuracy, Sensitivity, specificity, PPV and NPV are calculated for textural features such as Energy, correlation, contrast, variance and LOOP features. The classifiers 
Table 5. Evaluation of performance measures for various parameters using SVM, KNN, RF and DNN classifiers

\begin{tabular}{|c|c|c|c|c|c|c|c|}
\hline & Classifier & Energy & Contrast & Correlation & Variance & Loop & Hybrid \\
\hline \multirow{4}{*}{$\begin{array}{c}\text { Accuracy } \\
(\%)\end{array}$} & SVM & 56.80 & 58.67 & 55.53 & 50.53 & 50.00 & 50.00 \\
\hline & KNN & 54.00 & 52.67 & 48.73 & 58.07 & 90.73 & 91.07 \\
\hline & $\mathrm{RF}$ & 53.67 & 55.40 & 49.33 & 58.00 & 78.07 & 78.53 \\
\hline & DNN & 51.80 & 50.67 & 48.20 & 62.13 & 55.33 & 95.33 \\
\hline \multirow{4}{*}{$\begin{array}{c}\text { Sensitivity } \\
(\%)\end{array}$} & SVM & 59.44 & 57.75 & 57.28 & 50.53 & 36.00 & 38.00 \\
\hline & KNN & 52.69 & 51.91 & 49.18 & 55.72 & 86.58 & 87.13 \\
\hline & $\mathrm{RF}$ & 54.22 & 55.62 & 49.37 & 58.22 & 78.68 & 79.22 \\
\hline & DNN & 53.93 & 50.40 & 48.44 & 61.05 & 55.26 & 95.81 \\
\hline \multirow{4}{*}{$\begin{array}{c}\text { Specificity } \\
(\%)\end{array}$} & SVM & 51.47 & 66.27 & 52.40 & 50.80 & 72.00 & 76.00 \\
\hline & KNN & 76.93 & 79.07 & 77.07 & 79.47 & 92.73 & 93.73 \\
\hline & RF & 52.93 & 60.27 & 46.40 & 57.07 & 79.20 & 78.80 \\
\hline & DNN & 44.13 & 63.33 & 43.87 & 67.07 & 54.53 & 94.67 \\
\hline \multirow{4}{*}{$\begin{array}{l}\text { PPV } \\
(\%)\end{array}$} & SVM & 59.44 & 57.75 & 57.28 & 50.53 & 36.00 & 38.00 \\
\hline & KNN & 52.69 & 51.91 & 49.18 & 55.72 & 86.58 & 87.13 \\
\hline & $\mathrm{RF}$ & 54.22 & 55.62 & 49.37 & 58.22 & 78.68 & 79.22 \\
\hline & DNN & 53.93 & 50.40 & 48.44 & 61.05 & 54.26 & 94.81 \\
\hline \multirow{4}{*}{$\begin{array}{c}\text { NPV } \\
(\%)\end{array}$} & SVM & 55.93 & 61.59 & 54.63 & 50.50 & 14.00 & 12.00 \\
\hline & KNN & 59.39 & 53.58 & 47.46 & 66.25 & 93.50 & 92.56 \\
\hline & $\mathrm{RF}$ & 53.80 & 55.43 & 49.23 & 58.58 & 79.58 & 79.77 \\
\hline & DNN & 50.73 & 50.97 & 47.36 & 64.06 & 56.59 & 94.55 \\
\hline
\end{tabular}

such as SVM, KNN, and RF are compared with the proposed DNN classifier in terms of parameters is shown in Table 5. The Haralick and LOOP features are combined to form a hybrid features that shows better classification results for the proposed DNN classifier when compared with the existing classifiers.

The extraction of hybrid features achieved accuracy of $95.33 \%$ when compared with SVM, KNN, and Random Forest (RF) which have accuracy of $50.00 \%, 91.07 \%, 78.53 \%$ respectively. The comparison of the performance evaluation for the proposed and the existing methods by means accuracy of hybrid and other features are presented in the Fig. 7.

The extraction of hybrid features from the masking region achieved best sensitivity of $95.81 \%$ when compared with SVM, KNN, and RF obtained sensitivity of $38 \%, 87.13 \%$, and $79.22 \%$ respectively. The comparison of the performance evaluation for the proposed and the existing methods in terms sensitivity of hybrid features is shown in the Fig. 8.

The extraction of hybrid features from the masking region achieved best specificity of $94.67 \%$ when compared with SVM, KNN, and RF obtained specificity of $76 \%, 93.73 \%$, and $78.80 \%$ respectively. The comparison of the performance evaluation for the proposed and the existing methods in terms specificity of hybrid and other features are presented in the Fig. 9. The extraction of hybrid features from the masking region achieved best PPV of $94.81 \%$ when compared with SVM, KNN, and RF obtained PPV of $38 \%, 87.13 \%$, and $79.22 \%$ respectively. The comparison of the performance evaluation for the proposed and the existing methods in terms PPV of hybrid and the other features is presented in the Fig. 10. 


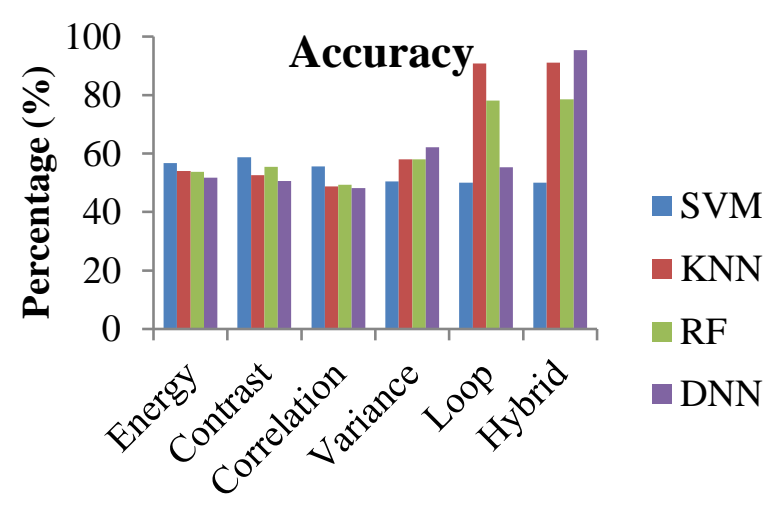

Parameters

Figure. 7 Comparison of parameters with respect to classifiers in terms of accuracy

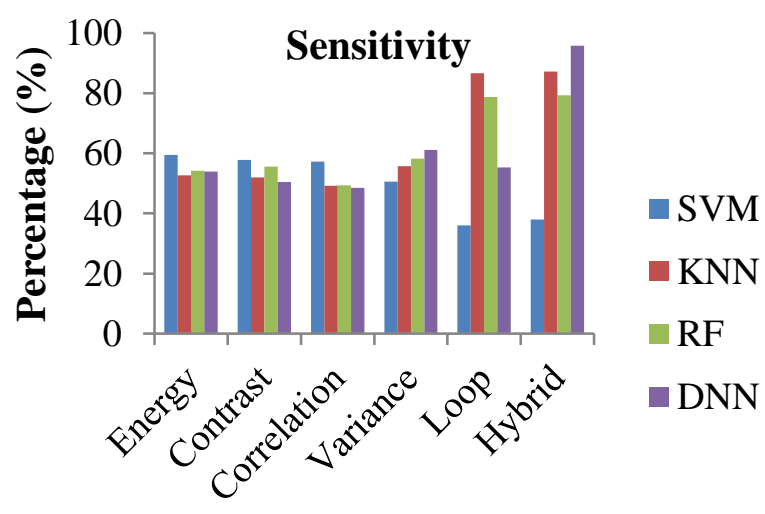

Parameters

Figure. 8 Comparison of parameters with respect to classifiers in terms of sensitivity

\section{Specificity}

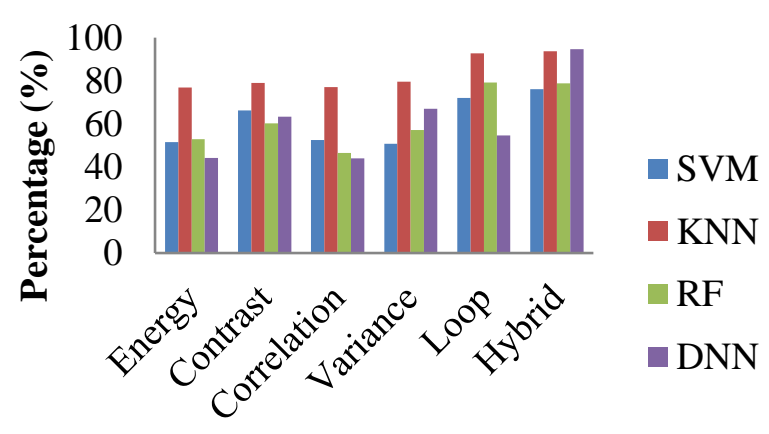

Parameters

Figure. 9 Comparison of parameters with respect to classifiers in terms of specificity

The extraction of hybrid features from the masking region achieved best NPV of $94.55 \%$ when compared with SVM, KNN, and RF obtained NPV of $12 \%, 92.56 \%$, and $79.77 \%$ respectively. The

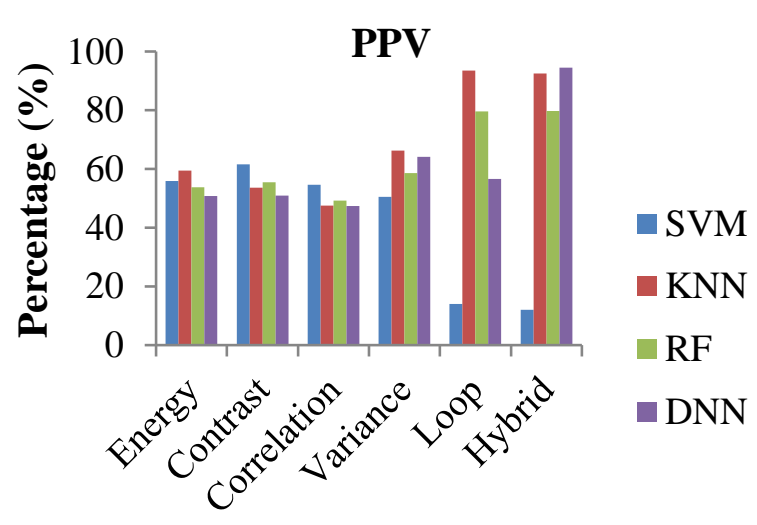

\section{Parameters}

Figure. 10 Comparison of parameters with respect to classifiers in terms of PPV

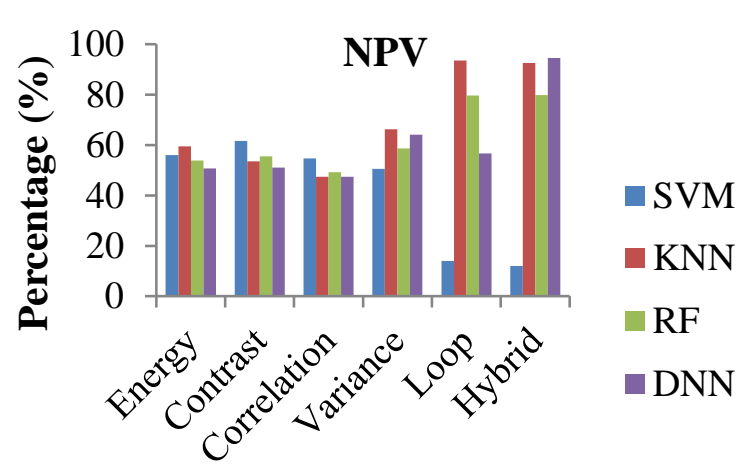

Parameters

Figure. 11 Comparison of parameters with respect to classifiers in terms of NPV

comparison of the performance evaluation for the proposed and the existing methods in terms NPV of hybrid and other features are shown in the Fig. 11.

From the results, it was clear that the proposed hybrid feature extraction showed better classification of Benign and Malignant MRI images using DNN. The hybrid features used in this research showed significant improvement in accuracy, which helps physicians to take accurate decisions on identifying cancer cells.

\subsection{Comparative analysis}

The proposed hybrid LOOP Haralick feature extraction method, namely Dynamic contrastenhancement technique [14] and K-NN [16] is compared with existing methods to analyze the performance in detection of breast cancer MRI. In the existing models the different feature extraction process includes extraction of GLCM and LDP 
Table 6. Comparison of the existing and proposed model with respect to the performance measures for the BIRADS dataset

\begin{tabular}{|c|c|c|}
\hline Authors & $\begin{array}{c}\text { Accuracy } \\
(\mathbf{\%})\end{array}$ & $\begin{array}{c}\text { PPV } \\
(\mathbf{\%})\end{array}$ \\
\hline $\begin{array}{c}\text { Gisela L. G. Menezes } \\
\text { (2016) [14] }\end{array}$ & 87 & 89 \\
\hline $\begin{array}{c}\text { Natascha C. D'Amico } \\
\text { (2020) [16] }\end{array}$ & 94 & 87 \\
\hline $\begin{array}{c}\text { Proposed hybrid LOOP } \\
\text { Haralick feature } \\
\text { extraction method }\end{array}$ & $\mathbf{9 5 . 3 3}$ & $\mathbf{9 4 . 8 1}$ \\
\hline
\end{tabular}

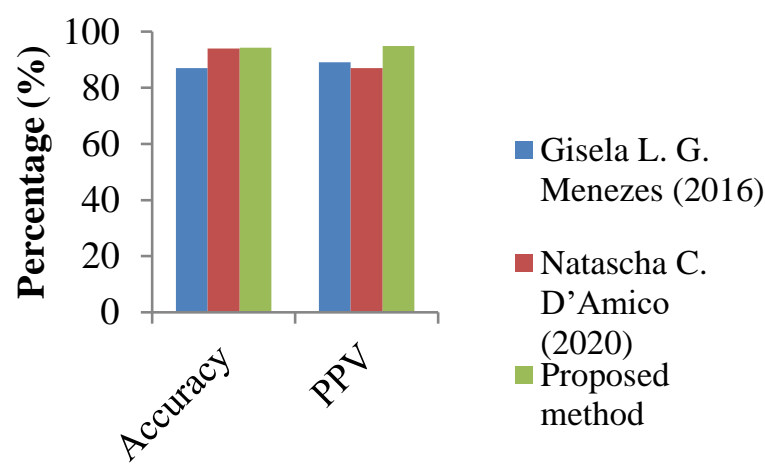

Performance Measure

Figure. 12 Comparison of accuracy and PPV measures for existing and proposed method

features. The comparison is made with respect to the common BI-RADS dataset used in the both existing and proposed method and it is presented in the Table 6. As [16] used more irrelevant or unnecessary 35 features that were extracted from the patients' dataset, the assessment of model reliability was weak and models were associated with a high risk of over fitting. This problem impacted on KNN during classification and lowered the value of accuracy to 94\%. To overcome such an issue, the proposed hybrid LOOP Haralick features included relevant or necessary features of 5 and were fed to SAE classifier obtained better accuracy of $95.33 \%$.

The Table 6 shows that the proposed hybrid LOOP Haralick features extraction is used for extracting features that does not create any complexity in the system when compared to the existing KNN models. The comparison graph of the existing and the proposed method is presented in the Fig. 12.

\section{Conclusion}

Breast cancer is the second leading cause of cancer death in women, so proper diagnosis is required to identify and control the spread of cancer cells. The MRI scanning is commonly recommended diagnosis technique for the women who are severely suffering from the breast cancer. But, the calcium components present in the breast are not diagnosed properly in MRI scans which leads to misclassification of breast cancer. This research proposed hybrid LOOP Haralick feature extraction technique to overcome the misclassification issues in MRI. Initially, CLAHE, Morphological and Multilevel Otsu's Thresholding and region growing techniques were performed to remove unwanted noise and also masking unwanted breast tissues in MRI. At last, hybrid parameters were obtained by combining Haralick texture feature, such as contrast, energy, correlation with LOOP features. The classification is performed based on the hybrid parameters using SAE to classify the breast MRI image as a Malignant or Benign. The performance of proposed hybrid LOOP Haralick feature showed improvement in Accuracy, Specificity, Sensitivity, PPV and NPV when compared to existing methods such as K-NN and Dynamic contrast-enhancement technique. The proposed hybrid LOOP Haralick feature extraction and existing methods such as K$\mathrm{NN}$ and Dynamic contrast-enhancement technique are compared using a common database known as BIRADS. The results of proposed hybrid LOOP Haralick feature method show significant improvement in accuracy of $3.83 \%$, when compared with the existing methods. However, the hybrid features were used in the proposed method showed complexity in the system due to the optimization problems. In the future, optimal features can be used in hybrid feature extraction to overcome optimization problems.

\section{Conflicts of Interest}

The authors declare no conflict of interest.

\section{Author Contributions}

The paper conceptualization, methodology, software, validation, have been done by $1^{\text {st }}$ author formal analysis, investigation, resources, data curation have been done by $2^{\text {nd }}$ author writingoriginal draft preparation, writing - review and editing, visualization, have been done by $3^{\text {rd }}$ author. The supervision and project administration, have been done by $4^{\text {th }}$ author.

\section{References}

[1] T. Almerey, D. Villacreses Z. Li, B. Patel, M. McDonough, T. Gibson, S. Maimone, R. Gray, 
and S. A. McLaughlin, "Value of Axillary Ultrasound after Negative Axillary MRI for Evaluating Nodal Status in High-Risk Breast Cancer", Journal of the American College of Surgeons, Vol. 228, No. 5, pp. 792-797, 2019.

[2] R. M. Mann, C. K. Kuhl, and L. Moy, "Contrastenhanced MRI for breast cancer screening", Journal of Magnetic Resonance Imaging, Vol. 50, No. 2, pp. 377-390, 2019.

[3] N. L. Eun, E. J. Son, H. M. Gweon, J. A. Kim, and J. H. Youk, "Prediction of axillary response by monitoring with ultrasound and MRI during and after neoadjuvant chemotherapy in breast cancer patients", European Radiology, Vol. 30, No. 3, pp. 1460-1469, 2020.

[4] S. Al-Hattali, S. J. Vinnicombe, N. M. Gowdh, A. Evans, S. Armstrong, D. Adamson, C. A. Purdie, and E. J. Macaskill, "Breast MRI and tumour biology predict axillary lymph node response to neoadjuvant chemotherapy for breast cancer", Cancer Imaging, Vol. 19, No. 1, pp. 91, 2019.

[5] S. Joukainen, A. Masarwah, M. Könönen, M. Husso, A. Sutela, V. Kärjä, R. Vanninen, and M. Sudah, "Feasibility of mapping breast cancer with supine breast MRI in patients scheduled for oncoplastic surgery", European radiology, Vol. 29, No. 3, pp. 1435-1443, 2019.

[6] D. Sheth and M. L. Giger, "Artificial intelligence in the interpretation of breast cancer on MRI", Journal of Magnetic Resonance Imaging, Vol. 51, No. 5, pp. 1310-1324, 2020.

[7] M. A. Mazurowski, A. Saha, M. R. Harowicz, E. H. Cain, J. R. Marks, and P. K. Marcom, "Association of distant recurrence-free survival with algorithmically extracted MRI characteristics in breast cancer", Journal of Magnetic Resonance Imaging, Vol. 49, No. 7, pp. e231-e240, 2019.

[8] A. Raghavendra, J. Wecsler, L. Ji, P. Sheth, C. Ricker, T. Church, R. Sposto, J. Lang, S. Sener, L. Larsen, and D. Tripathy, "Clinical findings and outcomes of MRI staging of breast cancer in a diverse population", Breast Cancer Research and Treatment, Vol. 174, No. 2, pp. 315-324, 2019.

[9] D. A. Sippo, G. I. Warden, K. P. Andriole, R. Lacson, I. Ikuta, R. L. Birdwell, and R. Khorasani, "Automated extraction of BI-RADS final assessment categories from radiology reports with natural language processing", Journal of Digital Imaging, Vol. 26, No. 5, pp. 989-994, 2013.

[10] A. Gubern-Mérida, R. Martí, J. Melendez, J. L. Hauth, R. M. Mann, N. Karssemeijer, and B.
Platel, "Automated localization of breast cancer in DCE-MR", Medical Image Analysis, Vol. 20, No. 1, pp. 265-274, 2015.

[11] G. J. Wengert, F. Pipan, J. Almohanna, H. Bickel, S. Polanec, P. Kapetas, P. Clauser, K. Pinker, T. H. Helbich, and P. A. T. Baltzer, "Impact of the Kaiser score on clinical decision-making in BIRADS 4 mammographic calcifications examined with breast MRI", European Radiology, Vol. 30, No. 3, pp. 1451-1459,2020.

[12] Y. Ji, H. Li, A.V. Edwards, J. Papaioannou, W. Ma, P. Liu, and M. L. Giger, "Independent validation of machine learning in diagnosing breast Cancer on magnetic resonance imaging within a single institution", Cancer Imaging, Vol. 19, No. 1, p. 64, 2019.

[13] M. Lunkiewicz, S. Forte, B. Freiwald, G. Singer, C. Leo, and R. A. Kubik-Huch, "Inter observer variability and likelihood of malignancy for fifth edition BI-RADS MRI descriptors in non-mass breast lesions", European Radiology, Vol. 30, No. 1, pp. 77-86,2020.

[14] G. L. Menezes, B. L. Stehouwer, D. W. Klomp, T. A. van der Velden, M. A. van den Bosch, F. M. Knuttel, V. O. Boer, van der W. J. Kemp, P. R. Luijten, and W. B. Veldhuis, "Dynamic contrastenhanced breast MRI at 7T and 3T: an intraindividual comparison study", SpringerPlus, Vol. 5, No. 1, p. 13, 2016.

[15] H. M. Gweon, N. Cho, S. Y. Kim, H. R. Koo, M. Seo, A. Chu, and E. J. Son, "Management for BIRADS category 3 lesions detected in preoperative breast MR imaging of breast cancer patients", European Radiology, Vol. 27, No. 8, pp. 3211-3216, 2017.

[16] N. C. D' Amico, E. Grossi, G. Valbusa, F. Rigiroli, B. Colombo, M. Buscema, D. Fazzini, M. Ali, A. Malasevschi, G. Cornalba, and S. Papa, "A machine learning approach for differentiating malignant from benign enhancing foci on breast MRI", European Radiology Experimental, Vol. 4, No. 1, p. 5, 2020.

[17] E. S. Burnside, E. A. Sickles, L. W. Bassett, D. L. Rubin, C. H. Lee, D. M. Ikeda, E. B. Mendelson, P. A. Wilcox, P. F. Butler, and C. J. D'Orsi, "The ACR BI-RADS ${ }^{\circledR}$ experience: learning from history", Journal of the American College of Radiology, Vol. 6, No. 12, pp. 851-860, 2009. 